\title{
Impact of the COVID-19 pandemic on gastrointestinal endoscopy activity in France
}

Authors

Arthur Belle ${ }^{*}, 1$, Maximilien Barret ${ }^{*},{ }^{1,2}$, David Bernardini ${ }^{3}$, Anne-Laure Tarrerias ${ }^{4}$, Erwan Bories ${ }^{5}$, Vianna Costil $^{6}$, Bernard Denis ${ }^{7}$, Rodica Gincul ${ }^{8}$, David Karsenti ${ }^{9}$, Stephane Koch ${ }^{10}$, Arthur Laquiere ${ }^{11}$, Thierry Lecomte ${ }^{12}$, Vincent Quentin ${ }^{13}$, Gabriel Rahmi ${ }^{14}$, Michel Robaszkiewicz ${ }^{15}$, Eric Vaillant ${ }^{16}$, Geoffroy Vanbiervliet ${ }^{17}$, Ariane Vienne ${ }^{18}$, Franck Dumeiran ${ }^{19}$, Olivier Gronier ${ }^{20}$, Stanislas Chaussade ${ }^{1,2}$, for the French Society of Digestive Endoscopy (Société Française d'Endoscopie Digestive)

Institutions

1 Gastroenterology and Digestive Oncology, Cochin Hospital, Assistance Publique-Hôpitaux de Paris, Paris, France

2 University of Paris, Paris, France

3 Gastroenterology Department, La Casamance Private Hospital, Aubagne, France

4 Gastroenterology Department, Hôpital Foch, Suresnes, Île-de-France, France

5 Gastroenterology Department, Hôpital Privé de Provence, Aix En Provence, France

6 Gastroenterology Department, Pôle Santé des 4 Temps, Puteaux, France

7 Médecine A, Hôpital Pasteur, Colmar, France

8 Service de Gastroentérologie, Hôpital Edouard Herriot, Lyon, France

9 Digestive Endoscopy Unit, Clinique de Paris-Bercy, Charenton-le-Pont, France

10 Gastroenterology Department, CHU Besançon, Besançon, France

11 Gastroenterology Department, Hôpital St Joseph, Marseille, France

12 Department of Hepatogastroenterology and Digestive Oncology, Trousseau University Hospital, Tours, France

13 Digestive Endoscopy Unit, Hôpital St Brieuc, St Brieuc, France

14 Gastroenterology and Digestive Endoscopy, Georges Pompidou European Hospital, Paris, France

15 Department of Gastrointestinal Endoscopy, CHRU de Brest, Brest, France

16 Department of Gastroenterology, Centre médical du Nord, Lille, France

17 Digestive Endoscopy, Hôpital de L'Archet 2, Nice, France

18 Department of Gastroenterology, Hôpital privé d'Anthony, Anthony, France

19 Gastroenterology Department, Société française d'endoscopie digestive, Limoges, France

20 Department of Gastroenterology and Hepatology, Clinique Sainte Barbe, Strasbourg, France submitted 28.4.2020

accepted after revision 17.6 .2020

published online 26.10 .2020

Bibliography

Endoscopy 2020; 52: 1111-1115

DOI 10.1055/a-1201-9618

ISSN 0013-726X

(c) 2020. Thieme. All rights reserved.

Georg Thieme Verlag KG, Rüdigerstraße 14,

70469 Stuttgart, Germany

Corresponding author

Stanislas Chaussade, MD, PhD, Gastroenterology and

Digestive Oncology, Cochin Hospital, 27 rue du Faubourg

St Jacques, 75014 Paris, France

stanislas.chaussade@aphp.fr

$\circledast$ Supplementary material

Online content viewable at:

https://doi.org/10.1055/a-1201-9618

\# Scan this QR-Code for the author commentary.

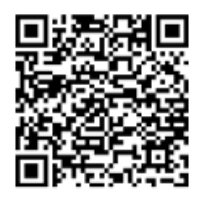

\section{ABSTRACT}

Background The SARS-CoV-2 pandemic has majorly affected medical activity around the world. We sought to measure the impact of the COVID-19 pandemic on gastrointestinal (GI) endoscopy activity in France.

Methods We performed a web-based survey, including 35 questions on the responders and their endoscopic practice, from 23 March to 27 March 2020, sent to the 3300 French gastroenterologists practicing endoscopy.

Results $694 \mathrm{Gl}$ endoscopists (21\%) provided analyzable data; of these, $29.4 \%$ (204/694) were involved in the management of COVID-19 patients outside the endoscopy department. During the study period, $98.7 \%$ (685/694) of

\footnotetext{
* Contributed equally to this article
} 
endoscopists had had to cancel procedures. There were 89 gastroenterologists $(12.8 \%)$ who reported symptoms compatible with COVID-19 infection, and a positive PCR test was recorded in $12 / 197$ (6.1\%) vs. $3 / 497$ (0.6\%) endoscopists in the high vs. low prevalence areas, respectively $(P$ $<0.001)$.
Conclusions The COVID-19 pandemic led to a major reduction in the volume of $\mathrm{Gl}$ endoscopies performed in France in March 2020. The prolonged limited access to GI endoscopy could lead to a delay in the management of patients with $\mathrm{Gl}$ cancers.

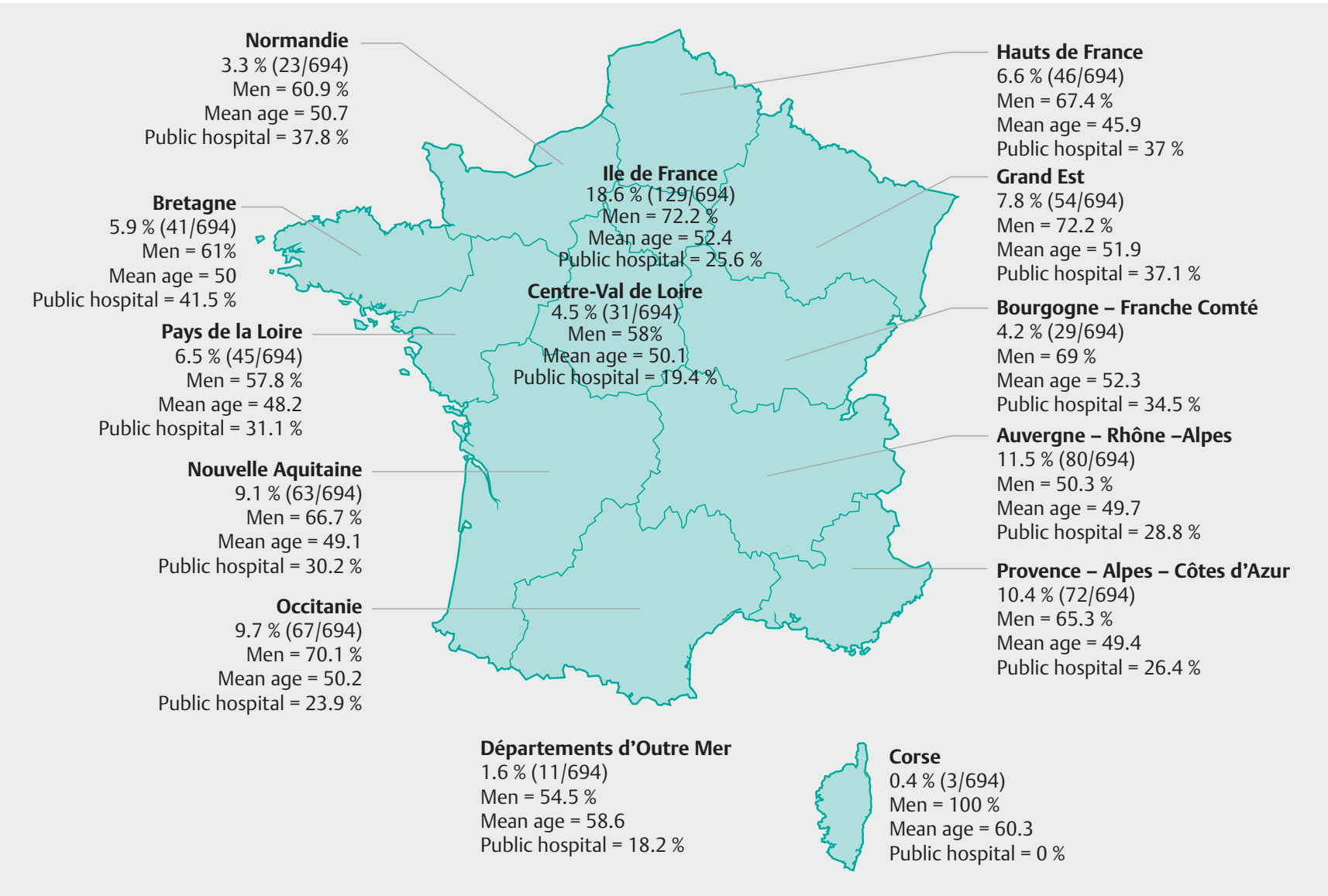

Fig. 1 Regions of activity and demographic characteristics of the responders.

\section{Introduction}

COVID-19 is thought to spread primarily through droplets of saliva, but airborne transmission and fecal excretion have been documented [1,2]. Healthcare professionals in endoscopy are exposed to COVID-19 by the contact of saliva droplets with their face and airways, touch contamination, and contact with patient stools [3,4]. Periendoscopic aerosolized infections have also been reported, placing upper gastrointestinal $(\mathrm{Gl})$ endoscopy among the major aerosol-generating procedures [5]. In addition, the high numbers of patients and accompanying persons visiting an endoscopy unit each day further increases the risk of contaminating the staff.

The French Society of Digestive Endoscopy (Société Française d'Endoscopie Digestive; SFED) and the European Society of Gastrointestinal Endoscopy (ESGE) had both published posi- tion statements by mid-April 2020 on the measures recommended to protect both patients and the endoscopy staff $[3,6]$. However, COVID-19 eventually spread massively in France, reaching over 180000 confirmed cases, including endoscopy patients and healthcare practitioners.

We sought to measure the impact of the COVID-19 pandemic on Gl endoscopy activity in France and to determine the risk of contamination of endoscopists at the peak of the epidemic.

\section{Methods}

We performed a web-based survey using an online questionnaire hosted on the SFED website. This survey included 35 questions on the description of the responders and their endoscopic practice (Appendix $\mathbf{1 s}$, available in online-online Sup- 
- Table 1 Impact of the COVID-19 pandemic on gastrointestinal endoscopy activity in France and comparison between the responses from those in the highest COVID-19 prevalence areas (North-East of France and Paris area) and those in the rest of the territory.

\begin{tabular}{|c|c|c|c|c|}
\hline & $\begin{array}{l}\text { Whole of France } \\
(n=694)\end{array}$ & $\begin{array}{l}\text { Highest COVID-19 } \\
\text { prevalence areas } \\
(n=197)\end{array}$ & $\begin{array}{l}\text { Lowest COVID-19 } \\
\text { prevalence areas } \\
(n=497)\end{array}$ & $P$ value \\
\hline Hospital's emergency plan been triggered, n (\%) & $547 / 608(90.0)$ & $163 / 172(94.8)$ & $384 / 436(88.1)$ & 0.02 \\
\hline Hospital had admitted COVID-19 patients, n (\%) & $505 / 694(72.8)$ & $156 / 197(79.2)$ & $349 / 497(70.2)$ & 0.02 \\
\hline $\begin{array}{l}\text { Involvement of the gastroenterologist in the management } \\
\text { of COVID-19 patients, } \mathrm{n}(\%)\end{array}$ & $204 / 694(29.4)$ & $88 / 197(44.7)$ & $116 / 497(23.3)$ & $<0.001$ \\
\hline Consultations had been canceled, $\mathrm{n}(\%)$ & $660 / 694(95.1)$ & 179/197 (90.9) & $481 / 497(96.8)$ & 0.003 \\
\hline Endoscopies had been canceled, $n$ (\%) & $685 / 694(98.7)$ & 193/197 (98.0) & $492 / 497(99.0)$ & 0.30 \\
\hline Endoscopy outpatient clinic closed, n (\%) & $511 / 694(73.6)$ & $155 / 197(78.7)$ & $356 / 497(71.6)$ & 0.07 \\
\hline $\begin{array}{l}\text { Endoscopy procedures still possible for hospital inpatients, } \\
\mathrm{n}(\%)\end{array}$ & $342 / 694(49.3)$ & $54 / 197(27.4)$ & $288 / 497(58.0)$ & $<0.001$ \\
\hline $\begin{array}{l}\text { Endoscopy procedures still possible for standard emergencies, } \\
\mathrm{n}(\%)\end{array}$ & 499/694 (71.9) & $114 / 197(57.9)$ & $385 / 497(77.5)$ & $<0.001$ \\
\hline Endoscopy procedures still possible for vital emergencies, n (\%) & $662 / 694(95.4)$ & $183 / 197(92.9)$ & $479 / 497(96.4)$ & 0.07 \\
\hline Endoscopy procedures performed for COVID-19 patients, n (\%) & $65 / 694(9.4)$ & $35 / 197(17.8)$ & $30 / 497(6.0)$ & $<0.001$ \\
\hline $\begin{array}{l}\text { Mean (SD) number of endoscopy procedures for COVID-19 } \\
\text { patients }\end{array}$ & $2.3(0.7)$ & $2.4(0.5)$ & $2.2(0.8)$ & 0.06 \\
\hline Specific circuit for COVID-19 patients, n (\%) & $305 / 694(43.9)$ & $75 / 197(38.1)$ & $230 / 497(46.3)$ & 0.05 \\
\hline Anesthesiologists or specialist nurses requisitioned, $n$ (\%) & $497 / 694(71.6)$ & $162 / 197(82.2)$ & $335 / 497(67.4)$ & $<0.001$ \\
\hline Mechanical ventilators requisitioned, n (\%) & $342 / 694(49.3)$ & $122 / 197(61.9)$ & $220 / 497(44.3)$ & $<0.001$ \\
\hline $\begin{array}{l}\text { Other materials from the endoscopy department requisitioned, } \\
\mathrm{n}(\%)\end{array}$ & 136/694 (19.6) & $64 / 197(32.5)$ & $72 / 497(14.5)$ & $<0.001$ \\
\hline $\begin{array}{l}\text { Endoscopy staff requisitioned for care of COVID-19 patients, } \\
n(\%)\end{array}$ & $454 / 694(65.4)$ & $156 / 197(79.2)$ & $298 / 497(60.0)$ & $<0.001$ \\
\hline
\end{tabular}

plementary material) from 23 March to 27 March 2020, 23 days after the 100th confirmed COVID-19 case. We sent an invitation to take the survey by email to the 3300 French gastroenterologists practicing endoscopy on 31 March, and follow-up emails on 2, 3, and 7 April 2020. We compared the data from the areas with the highest levels of COVID-19 (North and East of France, Paris area) to the rest of the country.

Data analysis was performed using GraphPad statistical software (La Jolla, California, USA). The mean (standard deviation [SD]) was used to describe variables with a normal distribution and the median (interquartile range $[I Q R]$ ) for those with a skewed distribution. Fisher's exact test was used to compare categorical data. Means were compared using an unpaired $t$ test. A $P$ value below 0.05 indicated statistical significance.

\section{Results}

\section{Characteristics of the responders}

There were $694 \mathrm{Gl}$ endoscopists (21.0\% of the 3300 gastroenterologists practicing endoscopy in France) who responded to the survey and provided analyzable data. The mean age of the study participants was 50.3 years, and $26.5 \%$ were 60 or older; $65 \%$ were men and $67 \%$ worked in a private hospital. Of the responders, $37.1 \%(258 / 694)$ worked in regions with a high COVID-19 prevalence (Hauts de France, Grand-Est, Bourgogne-Franche-Comté, lle de France). The regions of activity of all responders are shown in $\mathbf{F i g .} \mathbf{1 .}$

\section{Consequences for gastroenterology and endoscopy practice}

There were 547 responders (90.0\%) who reported that an emergency plan had been activated in their hospital, and $72.8 \%$ (505/694) had specific departments for COVID-19 patients in their center, with a mean of $54.5 \%$ of the beds requisitioned for COVID-19 patients. Mechanical ventilators and endoscopy staff had been requisitioned in $49.3 \%$ and $65.4 \%$ of centers, respectively. The majority of gastroenterologists participated in healthcare activities related to the COVID-19 pandemic: $15.3 \%$ (106/694) performed telephone consultation or triage of COVID-19 patients; $37.3 \%$ (259/694) did on-call duty outside the emergency department; $23.6 \%$ (164/694) did on-call duty in the emergency department; 
- Table 2 Impact of the COVID-19 pandemic on gastrointestinal endoscopy procedures in France and comparison between the responses of those in the highest COVID-19 prevalence areas (North-East of France and Paris area) and those in the rest of the territory.

\begin{tabular}{|c|c|c|c|c|}
\hline & $\begin{array}{l}\text { Whole of France } \\
(n=694)\end{array}$ & $\begin{array}{l}\text { Highest COVID-19 } \\
\text { prevalence areas } \\
(n=197)\end{array}$ & $\begin{array}{l}\text { Lowest COVID-19 } \\
\text { prevalence areas } \\
(n=497)\end{array}$ & $P$ value \\
\hline Endoscopic procedures performed without a mask, n (\%) & $394 / 694(56.8)$ & $114 / 197(57.9)$ & $280 / 497(56.3)$ & 0.70 \\
\hline Difficulty obtaining surgical masks for endoscopy, $\mathrm{n}(\%)$ & $97 / 694(14.0)$ & 23/197 (11.7) & $74 / 497(14.9)$ & 0.30 \\
\hline Difficulty obtaining FFP2 masks for endoscopy, n (\%) & $378 / 694(54.5)$ & $104 / 197(52.8)$ & $274 / 497(55.1)$ & 0.60 \\
\hline Difficulty obtaining eye protections for endoscopy, $\mathrm{n}(\%)$ & $173 / 694(24.9)$ & $31 / 197(15.7)$ & $142 / 497(28.6)$ & 0.004 \\
\hline Difficulty obtaining gowns for endoscopy, n (\%) & $131 / 694(18.9)$ & $32 / 197(16.2)$ & $99 / 497(19.9)$ & 0.30 \\
\hline Difficulty obtaining gloves for endoscopy, $\mathrm{n}(\%)$ & $10 / 694(1.4)$ & $1 / 197(0.5)$ & $9 / 497(1.8)$ & 0.30 \\
\hline Difficulty obtaining surgical cap for endoscopy, n (\%) & $19 / 694(2.7)$ & $5 / 197(2.5)$ & $14 / 497(2.8)$ & $>0.99$ \\
\hline Difficulty obtaining hydroalcoholic gel for endoscopy. n (\%) & $13 / 694(1.9)$ & $5 / 197(2.5)$ & $8 / 497(1.6)$ & 0.50 \\
\hline Gastroenterologist had COVID-19 symptoms, n (\%) & $89 / 694(12.8)$ & $42 / 197(21.3)$ & $47 / 497(9.5)$ & $<0.001$ \\
\hline COVID-19 infection documented by a positive PCR, n (\%) & $15 / 694(2.1)$ & $12 / 197(6.1)$ & $3 / 497(0.6)$ & $<0.001$ \\
\hline
\end{tabular}

and $13.8 \%(96 / 694)$ performed others activities. Besides endoscopy procedures, $29.4 \%$ (204/694) took care of COVID19 patients, accounting for $30.5 \%$ of their medical activity. Of the responders, $95.1 \%$ had had to cancel consultations, and $86.3 \%$ of all consultations had been canceled. Among the $57.4 \%$ of the responders providing telephone consultations, a mean of 11.7 phone consultations took place per day. Nevertheless, $32.1 \%$ of the phone consultations were ultimately cancelled because a clinical examination was needed.

The anesthesiologists usually dedicated to the endoscopy department had been requisitioned from the departments of $71.6 \%$ of responders, and $98.7 \%(685 / 694)$ of the gastroenterologists had had to cancel endoscopy procedures, with $91.2 \%$ of procedures being cancelled. An order of priority on the waiting list has been proposed in $87.8 \%$ of the cases. A total of $73.6 \%$ (511/694) of the endoscopy outpatient clinics were closed. Endoscopies could still be performed for patients admitted to the hospital by $49.3 \%$ of responders, for non-vital emergencies (e.g. foreign body removal, Gl bleeding without hemodynamic instability, caustic ingestion) by $71.9 \%$ of responders, and for vital emergencies by $95.4 \%$. Only $9.4 \%$ of responders eventually performed endoscopies in COVID-19 patients, with a median (range) of $2.3(1-12)$ endoscopies during the week of the survey. A specific circuit for COVID-19 patients had been organized in $44 \%$ of the endoscopy departments.

\section{COVID-19 infection rates among gastroenterologists and implementation of protective measures}

Of the responders, $56.8 \%$ performed $\mathrm{Gl}$ endoscopy procedures without wearing a mask. The practitioners reported difficulty obtaining the following equipment for endoscopy: surgical mask (14.0\%), FFP2 mask (54.5\%), eye protection (24.9\%), gown (18.9\%), gloves (1.4\%), surgical cap (2.7\%), hydroalcoholic gel $(1.9 \%)$.
Symptoms compatible with COVID-19 infection were reported by $12.8 \%(89 / 694)$ of the gastroenterologists; of these, $37.1 \%$ (33/89) had a PCR test for COVID-19 performed, a mean of 6 days after the onset of symptoms, and the test was positive in $45.5 \%(15 / 33)$ of the tests. One responder had required hospital admission. Of those reporting symptoms, $43.8 \%(39 / 89)$ believed they had been contaminated at work.

- Table 1 and $>$ Table 2 show the comparative impact of the COVID-19 pandemic on GI endoscopy in France, and compare the highest prevalence areas (North-East of France and Paris area) with the rest of France.

\section{Discussion}

We conducted a survey among all French gastroenterologists practicing endoscopy, in a period of intense viral circulation in France, after there had been over 20000 documented COVID19 cases and before the effects of the lockdown (instituted on 17 March 2020) were observable. The 694 responders, accounting for $21 \%$ of the 3300 gastroenterologists in France, likely include a large proportion of the 806 active members of the French Society of Digestive Endoscopy registered for the year 2019.

Our data illustrate the major impact of the pandemic on $\mathrm{Gl}$ endoscopy activity in France, with a $91.2 \%$ reduction in procedure numbers. This reduction was the consequence of the requisition of the anesthesiology teams and their equipment, decided by the Ministry of Health, explaining why the endoscopy activity reduction affected equally the areas with high or low COVID-19 prevalence. Indeed, in France, over $90 \%$ of colonoscopies are performed with the patient under general anesthesia by an anesthesiologist or a specialist nurse [7]. While endoscopies for vital emergencies were still possible in virtually all cases, endoscopies for most emergencies and for inpatients were reported as being impossible by $28.1 \%$ and $50.7 \%$ of re- 
sponders, respectively, with significant disparities over the territory.

We documented a $12.8 \%$ prevalence of COVID-19 symptoms among $\mathrm{Gl}$ endoscopists, significantly higher in the areas with higher COVID-19 prevalence. Of note, the difficulty accessing diagnostic tests in France at this time explains why only a third of the gastroenterologists who were symptomatic had a PCR test performed. Our infection rate is higher than the $4.3 \%$ (confirmed) infection rate reported in Northern Italy [4], and is possibly explained by a high involvement of the gastroenterologists in the management of COVID-19 patients, and the use of personal protective equipment (PPE), such as masks, by less than half of the endoscopists. This last point could result both from a lack of awareness of the importance of wearing a mask when performing endoscopy procedures, and a lack of optimal PPE (e.g. FFP2 masks) in over half of the endoscopy departments.

Eventually, lockdown on 17 March resulted in a decrease in the number of COVID-19 patients from 16 April, and the lockdown was stopped on 11 May 2020. However, the current endoscopy activity in France still does not exceed $80 \%$ of the actual capacity, because many anesthesiologists are still managing COVID-19 units, many patients are not willing to come to the hospital for non-emergent investigations, and protective measures limit the number of patients in the endoscopy outpatient clinics. Therefore, thousands of endoscopy procedures cancelled during the lockdown have still not been rescheduled. In the meantime, the improved knowledge about the virus, the first severe cases among endoscopists, and the improved availability of PPE, including FFP2 masks, seems to have resulted in an increased adherence to protective measures among endoscopists.

The limitations of our work include the limited number of responders, with an over-representation of private practitioners, and possible bias toward the limitation of endoscopy activity (because most public hospitals were able to maintain a minimal endoscopy activity throughout the epidemic). Second, only a small proportion of the responders with symptoms had diagnostic confirmation by PCR, and severe cases may have been overlooked because they would have been unable to take the survey. Finally, the short duration of the study period and the relative early phase in the pandemic may also lead to underestimation of certain events, such as the contamination rate of endoscopists or the difficulties in obtaining certain types of PPE.

In conclusion, we observed a $91.2 \%$ reduction of Gl endoscopy activity at the end of March 2020, resulting from the COVID-19 pandemic and the decisions of the healthcare authorities. While emergent endoscopies were still possible, even in the areas with the highest COVID-19 prevalence, the prolonged limited access to Gl endoscopy could lead to a delay in the management of thousands of patients with Gl cancers.

\section{Competing interests}

The authors declare that they have no conflict of interest.

\section{References}

[1] Tian Y, Rong L, Nian W et al. Review article: gastrointestinal features in COVID-19 and the possibility of faecal transmission. Aliment Pharmacol Ther 2020; 51: 843-851

[2] Wilson NM, Norton A, Young FP et al. Airborne transmission of severe acute respiratory syndrome coronavirus- 2 to healthcare workers: a narrative review. Anaesthesia 2020: doi:10.1111/anae.15093

[3] Gralnek IM, Hassan C, Beilenhoff $U$ et al. ESGE and ESGENA Position Statement on gastrointestinal endoscopy and the COVID-19 pandemic. Endoscopy 2020; 52: 483-490

[4] Repici A, Aragona G, Cengia G. Low risk of covid-19 transmission in Gl endoscopy. Gut 2020: doi:10.1136/gutjnl-2020-321341

[5] Parodi SM, Liu VX. From containment to mitigation of COVID-19 in the US. JAMA 2020: doi:10.1001/jama.2020.3882

[6] SFED. Epidémie de COVID-19 - Recommandations en endoscopie digestive. 11.03 2020: https://www.sfed.org/files/files/covid19endo_reco.pdf Accessed: 29 June 2020

[7] Barret M, Boustiere C, Canard J-M et al. Factors associated with adenoma detection rate and diagnosis of polyps and colorectal cancer during colonoscopy in France: results of a prospective, nationwide survey. PloS One 2013; 8: e68947 